\title{
Kundenzufriedenheitsanalyse - Bericht über ein studentisches Projekt
}

\author{
Michael Jastrob, Peter Jentsch, Stefan Müller
}

Kundenzufriedenheit nimmt eine zentrale Stellung im Bereich Marketing ein, da Unternehmen in zunehmendem Maße erkennen, dass ein enger Zusammenhang zwischen Kundenzufriedenheit, zukünftigem Kundenverhalten und langfristigem Geschäftserfolg besteht. Insbesondere durch den immer intensiver gewordenen globalen Wettbewerb entsteht ein Konkurrenzdruck, der die Unternehmen dazu zwingt, sich von den Wettbewerbern abzugrenzen. Im Produktbereich sind Qualitätssteigerungen oft nicht mehr ohne weiteres möglich. Somit legen Unternehmen den Schwerpunkt auf andere Leistungsparameter, um ihre Aktivitäten auf die Bedürfnisse der Kunden wirksamer auszurichten. Eine der Aufgaben des Marketings besteht darin, die Ursachen der Unzufriedenheit von Kunden aufzudecken. Daraus können Rüickschluisse gezogen werden, welche Auswirkungen das Kundenverhalten auf das Unternehmen selbst hat. Anzeichen von Unzufriedenheit beim Kunden gelten als Frühwarnsignale von Marktanteilsverlusten. Deshalb muss versucht werden, Problembereiche zu identifizieren und Verbesserungsmaßnahmen rechtzeitig durchzufuihren. Die Zeitdimension spielt bei der Kundenzufriedenheit eine besondere Rolle. Unternehmen sind bestrebt, ihre Kunden langfristig an sich zu binden. Kundenbindungsstrategien sind für das Unternehmen kostenguinstiger als Strategien zur Gewinnung von Neukunden oder zur Rückgewinnung von ehemaligen Kunden.

\section{Was ist Kundenzufriedenheit?}

Kundenzufriedenheit gilt allgemein als ein Ergebnis eines Vergleichsprozesses zwischen den Kundenerwartungen und den erhaltenen Leistungen. Diese Leistungen unterliegen einer subjektiven Wertung des Kunden. In dieser Wertung spielen auch aktuelle Erfahrungen des Kunden sowie die Lösung eines individuellen Problems eine wichtige Rolle. Beeinflussungsfaktoren sind u. a.: Erwartungen des Kunden; sein individuelles Anspruchsniveau innerhalb der aktuellen Nachfragesituation; das Image und Leistungsversprechen des Anbieters und mögliche Alternativen. Bewertet werden materielle Leistungsfaktoren (z. B. Qualität der Produkte) und immaterielle Leistungsparameter (z. B. Service, Beratung). Unternehmen sollten mittels eines Soll-Ist-Vergleichs versuchen, die Erwartungen des Kunden zu übertreffen, um das Zufriedenheitsurteil ihrer Kunden positiv ausfallen zu lassen und es langfristig zu festigen. In der einschlägigen Literatur erklären Autoren Zufriedenheit der Kunden mit Erfuillung der Kundenerwartungen. Folglich müssten sich Kunden unzufrieden fuihlen, wenn ihre Er- wartungen unterschritten wurden. U. E. führt erst eine erhebliche Diskrepanz zwischen Erwartungen und wahrgenommener Leistung zur Zufriedenheit im Falle einer positiven Abweichung und zur Unzufriedenheit im Falle einer negativen Abweichung. Werden die Erwartungen von Kunden erfüllt, führt dies lediglich zu einem Indifferenzgefühl. Grundlegend kann man von einer Vorstellung des Kunden über die gewünschte bzw. angemessene Leistung ausgehen. Die gewünschte Leistung spiegelt die Erwartungen des Kunden im Idealfall wider, während die angemessene Leistung lediglich eine vom Kunden akzeptierte Leistung darstellt. Dazwischen liegt eine Toleranzzone, in der der Kunde die Leistung als zufriedenstellend ansieht.

\section{Kundenzufriedenheit als Gewinn}

Eines der Hauptziele von Unternehmen muss die Erwirtschaftung von Gewinn sein. In der Industrie wird eine Erhöhung des Gewinns im Allgemeinen durch Rationalisierungsmaßnahmen und Verbesserung der Produktivität erreicht. Diese Faktoren sind im Dienstleistungssektor schwierig anwendbar und führen in der Dienstleistungsbranche oft zur Verschlechterung der Kundenzufriedenheit. Eine Kostenreduktion im Handel oder eine Erhöhung der Flächenrendite muss nicht automatisch eine Verbesserung der Gewinnsituation bedeuten. Diese Maßnahmen gehen häufig zu Lasten der customer satisfaction, da z. B. die Übersichtlichkeit der Verkaufseinrichtung für den Kunden verloren geht. Der daraus resultierende Rückgang der Kundentreue fuihrt zu einem Umsatzrückgang. Ist ein Dienstleistungsunternehmen zu sehr auf seine Effizienzerhöhung fixiert, gerät die customer satisfaction in den Hintergrund. Die Kundenzufriedenheit sollte aber ein Schlüsselfaktor sein, denn ein treuer Kunde ist bereit, einen „premium price“ zu zahlen. Dadurch kann das Unternehmen einen höheren Deckungsbeitrag erwirtschaften und somit seine Kosten besser decken. Wird der Kunde als Ressource betrachtet, steigt im Laufe der Zeit seine Ertragskraft - dies ist auf folgende Gründe zurückzuführen:

- Die Marketingkosten für die Gewinnung eines Neukunden stellen einen erheblich höheren Aufwand dar (z. B. Beratungsgespräche beim Erstkauf) als für die Festigung der Bindung eines treuen Kunden (coporate identity).

- Ein zufriedener Kunde tätigt nicht nur Wiederkäufe im Unternehmen, sondern wirbt auch für das Unternehmen, indem er seine Zufriedenheit anderen Menschen mitteilt. Im Gegensatz dazu äußert ein unzufriedener Kunde seine Meinung gegenüber anderen 
potenziellen Kunden im Durchschnitt 10 mal.

- Die Bedürfnisse eines Kunden können im Laufe einer Kundenbeziehung zunehmen (z. B. Ersatzteile, Einzelprodukte, Sortiment).

- Die Treue eines Kunden stellt für Wettbewerber oft eine unuiberwindbare Barriere dar, da Kundentreue neben der Verringerung der Marketingkosten auch zu einer Absatzsteigerung und einer Ertragserhöhung fuihrt.

Umgekehrt kann Kundenunzufriedenheit, die aus Produktnutzung und/oder Umfeld resultiert, beim Kunden verschiedene Reaktionen hervorrufen. Eine mögliche Reaktion ist die Abwanderung des Kunden. Hat der Kunde erst die Marke gewechselt, besteht fuir den Anbieter kaum eine Chance, sich mit der Unzufriedenheit und ihren Gründen auseinanderzusetzen, weil sich der Kunde nicht über seine Unzufriedenheit geäußert hat. Eine Abwanderung von unzufriedenen Kunden ist um so wahr- scheinlicher, je wettbewerbsintensiver das Umfeld ist. Eine negative „Mund-zu-Mund-Propaganda“ führt dazu, dass die Unzufriedenheit an einen weiten Personenkreis weitergeleitet wird und somit bestehende und potenzielle Kunden in ihrem Kaufverhalten beeinflusst werden können. Diese negative Einflussnahme kann weite Kreise ziehen und damit dem Anbieter enorme wirtschaftliche Schäden zufuigen.

Die Kundenbindung sowie die Gestaltung des Kundenwertes (customer value) stellt für ein Dienstleistungsunternehmen folglich die letztlich eigentliche Hauptquelle des Gewinns dar.

\section{Kundenzufriedenheitsanalyse bei der MEGA Betriebs GmbH}

Im Rahmen unserer Ausbildung zu Wirtschaftsingenieuren haben wir im Wintersemester 1999/2000 u. a. eine

\begin{tabular}{|c|c|}
\hline \multirow{3}{*}{$\begin{array}{l}\text { 1. Welcher Kundenkreis ist är uns } \\
\text { interessant und erreichbar? }\end{array}$} & Definieren der Zelgruppe(n) \\
\hline & $\begin{array}{l}\text { - Kaufkunden } \\
\text { - Besucher IInteressenten }\end{array}$ \\
\hline & $\frac{1}{7}$ \\
\hline \multirow{3}{*}{$\begin{array}{l}\text { 2. Welche Branche verfügt über einen } \\
\text { hohen Kundenstrom und } \\
\text { repräsentativen Be wölkerungs- } \\
\text { querschnitt? }\end{array}$} & Festlegung der zu untersuchenden B ranche \\
\hline & - Möbeleinzelhandel \\
\hline & 7 \\
\hline \multirow{3}{*}{$\begin{array}{l}\text { 3. Mit welcher Methode mercen die } \\
\text { Daten erfaßst? }\end{array}$} & Auswahl der Urtersuchungsmethode \\
\hline & $\begin{array}{l}\text { - Interview } \\
\text { - Totalerhebung }\end{array}$ \\
\hline & $\frac{1}{7}$ \\
\hline \multirow{4}{*}{$\begin{array}{l}\text { 4. Welche Infomationen sind zur } \\
\text { Analyse und Auswertung } \\
\text { wünschenswert? }\end{array}$} & Fragebogenerstellung \\
\hline & $\begin{array}{l}\text { - demographische Fragen } \\
\text { - Verkaufseinichtung } \\
\text { - Produktangebot } \\
\text { - Verkaufspersonal } \\
\text { - Werbeprospekte } \\
\text { - Lieferung / Versand } \\
\text { - etc. }\end{array}$ \\
\hline & $\begin{array}{l}\text { Wissenschaftliche Validierung } \\
\text { Nutzen der Infomation, Aussagegehalt, } \\
\text { Wichtigkeit, Korrelationstähigkeit ) }\end{array}$ \\
\hline & 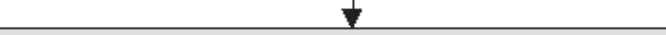 \\
\hline \multirow{4}{*}{$\begin{array}{l}\text { 5. Welcher Partner entspricht unseren } \\
\text { Ermartungen am besten? } \\
\text { (siehe Frage 2) }\end{array}$} & Auswahl des geeigneten $P$ artners $\rightarrow$ ME GA Möbel \\
\hline & $\checkmark$ \\
\hline & $\begin{array}{l}\text { kundenspezifische } \\
\text { Validierung * }\end{array}$ \\
\hline & 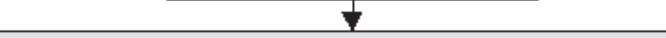 \\
\hline \multirow{2}{*}{$\begin{array}{l}\text { 6. We erreichen wr unsere vakanten } \\
\text { Zielgruppen? }\end{array}$} & Durchfuihrung der Befragung \\
\hline & point of sale and delivery \\
\hline \multirow{3}{*}{$\begin{array}{l}\text { 7. We erfolgt die Aufbereitung und } \\
\text { DVtechnische Erfassung der } \\
\text { Daten? }\end{array}$} & Datenerfassung \\
\hline & $\begin{array}{l}\text { - Codierung der Antworten } \\
\text { - Aufbau einer Datenbank unter MS-Excel } \\
\text { - Dateneingabe unter Vermendung der festgelegten code keys }\end{array}$ \\
\hline & 7 \\
\hline \multirow{3}{*}{$\begin{array}{l}\text { 8. Welche Ergebnisse können aus den } \\
\text { worhandenen Infomationen gefitert } \\
\text { werden? }\end{array}$} & Analyse und Auswertung \\
\hline & $\begin{array}{l}\text { - gezielte Ausmahl vermertbarer Informationen } \\
\text { - Bilden aussagefähiger korrelativer Verknüpfungen } \\
\text { - graphische Darstellung der Analyseergebnisse }\end{array}$ \\
\hline & $\frac{1}{7}$ \\
\hline \multirow{2}{*}{$\begin{array}{l}\text { 9. Wie können die Analyseergeknisse } \\
\text { bewertet werden? }\end{array}$} & Bewertung \\
\hline & $\begin{array}{l}\text { - Überprüfung der Repräsentaivität } \\
\text { (Einbeziehung won Globalaussagen statistischer ämter) } \\
\text { - Schlußłolgenungen bezüglich der Kundenzutiedenheit bei MEGA-Möbel }\end{array}$ \\
\hline
\end{tabular}

*) nach der kundenspezifischen $V$ alidierung sollte ein Pre-Test durchgeführt werden, um eine $V$ erifizierung des Fragebogens vornehm en zu können (B eseitigung inhaltlicher Mängel und nicht schlüssig gestellter Fragen)

Abb. 1: Ablaufschema der Kundenzufriedenheitsanalyse bei der MEGA Betriebs GmbH
Projektarbeit durchgefuihrt. In den Lehrfächern „Absatzwirtschaft“ und „Kundenservice“ hatten wir viel über Kundenzufriedenheit und Probleme der Messung von Kundenzufriedenheit schlag unseres Dozenten, Herrn Dr. Dr. Heyer auf, eine Kundenzufriedenheitsanalyse praktisch durchzufuihren, um so die dabei entstehenden Probleme und Möglichkeiten ihrer Lösung „am eigenen Leib“ zu erleben. Nach ersten Diskussionen über konzeptionelle Fragen der Herangehensweise und Durchfuihrung grenzten wir den Kreis von Unternehmen ein, die aus unserer Sicht für eine Kundenzufriedenheitsanalyse in Frage kämen. Die ersten Versuche der Kontaktaufnahme mit Unternehmen waren fuir uns interessant und lehrreich zugleich. Um die Bedeutung von Kunden für Unternehmen wissend waren wir davon ausgegangen, dass sich ein(e) Geschäftsfuihrer(in) eines Unternehmens über unser Vorhaben freuen würde - aber die Wirklichkeit hatte uns bald eingeholt. Bei einigen kontaktierten Unternehmen hatten wir den Eindruck, dass sie die Befindlichkeiten ihrer Kunden nicht sonderlich interessant finden, andere Unternehmen hatten wohl Sorge, dass wir den „geregelten Betriebsablauf “ stören könnten. Um so erfreulicher war es für uns, dass die MEGA Betriebs $\mathrm{GmbH}$, Filiale Wildau, und insbesondere ihr Geschäftsfuihrer, Herr Früichtenich, unsere Absicht aufgriff und tatkräftig unterstiitzte. Unsere Herangehensweise und Durchfuihrung der Kundenzufriedenheitsanalyse bei der MEGA Betrieb $\mathrm{GmbH}$ ist nebenstehendem Ablaufschema zu entnehmen. diskutiert. Schnell griffen wir den Vor- 


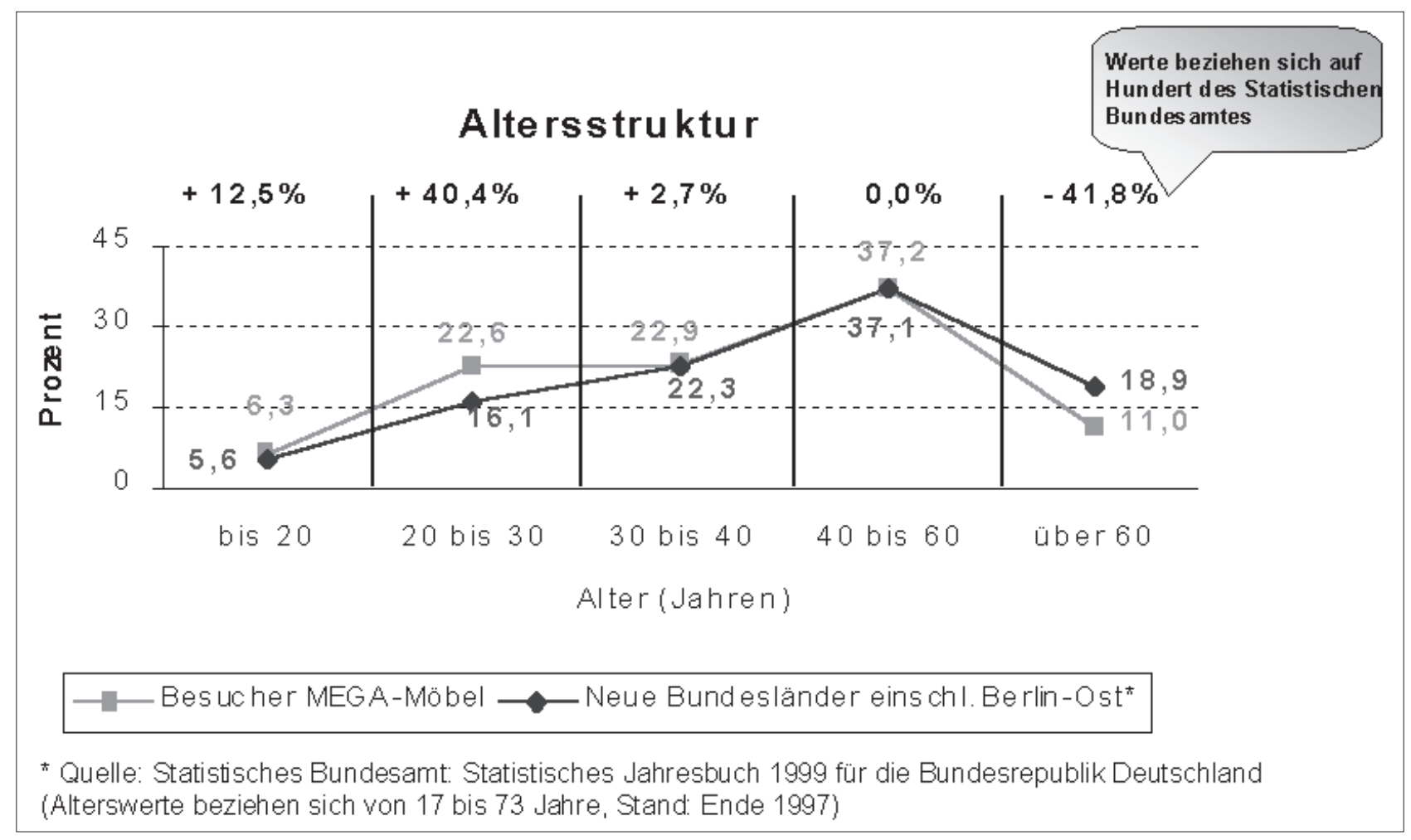

Abb. 2: Vergleich der Altersstruktur Besucher MEGA-Möbel und Neue Bundesländer

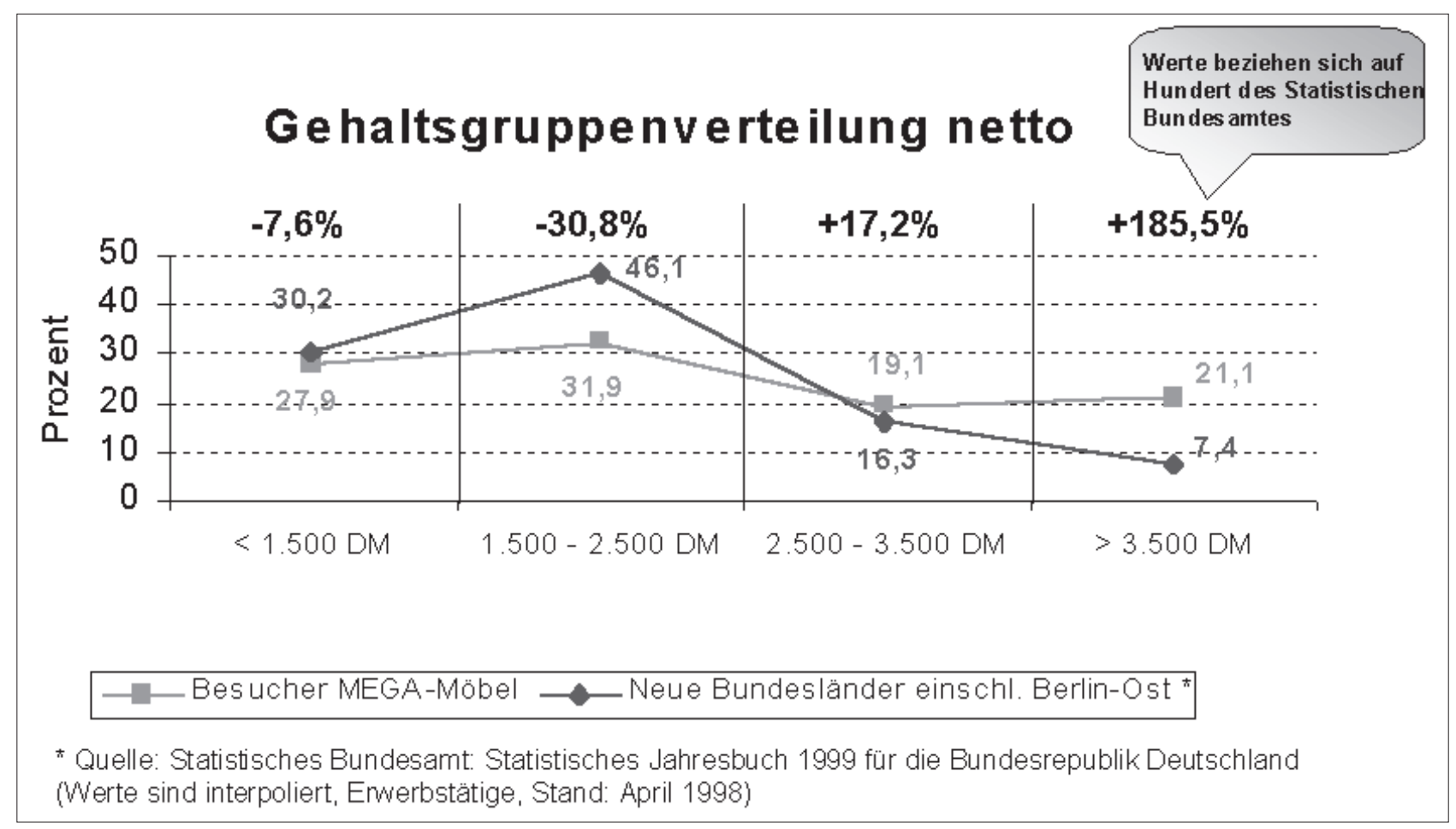

Abb. 3: Vergleich der Nettogehaltsgruppenverteilung Besucher MEGA-Möbel und Neue Bundesländer

\section{Ergebnisse der Kundenzufriedenheitsanalyse}

Die Befragung der Besucher von MEGA-Möbel erfolgte an 14 Terminen im November 1999. Die Zeitdauer je Befragungstag betrug im Durchschnitt ca. 6 Stunden. In dieser Zeit wurden insgesamt 883 Personen befragt. Davon erhielten wir 520 Ablehnungen (58,8 \%) und von 363 Personen (41,1\%) ausgefüllte Fragebögen. Von den 363 Personen gaben 93 an, dass sie am Befragungstag Einkäufe bei MEGA-Möbel tätigten. Von den 883 befragten Personen waren 174 Erstbesucher (19,7\%).
Im ersten Schritt unserer Untersuchung analysierten wir die demografische Zusammensetzung der interviewten Personen.

Von der Altersstruktur ausgehend ist eine aussagefähige Repräsentativität gegeben. Der starke Unterschied in der Gruppe der 20- bis 30-jährigen zwischen der Altersstruktur von MEGA-Möbel und der Neuen Bundesländer einschl. Berlin-Ost $(+40,4 \%)$ ist darauf zurückzuführen, dass die Bereitschaft dieser Gruppe, den 
Fragebogen auszufüllen, am größten war. In der Gruppe der über 60-jährigen ist der Unterschied deshalb so groß $(-41,8 \%)$, da die Mobilität dieser Leute teilweise stark eingeschränkt ist.

Unter Zugrundelage der Nettogehaltsstruktur der Besucher von MEGA-Möbel ergibt sich keine Repräsentativität, denn die Unterschiede zu der Struktur der Neuen Bundesländer einschl. Berlin-Ost sind zu eklatant. Vor allem der Unterschied in der Nettogehaltsgruppe $>3.500$ DM mit +185,5 \% gegenüber den Neuen Bundesländern ist zu hoch, auch wenn die Zielgruppe von MEGA-Möbel die „Besserverdienenden“ sein sollte.

Darüber hinaus untersuchten wir folgende Punkte:

- Geschlechterverteilung,

- Wohnort,

- Anzahl der Besuche und der Käufe bei MEGA-Möbel

- Internetnutzung bzgl. Informationsbeschaffung und E-Commerce.

Im nachfolgenden Schritt untersuchten wir die Zufriedenheit bei den Kriterien, die sich auf MEGA-Möbel beziehen:

- Verkaufseinrichtung,

- Angebot,

- Personal,

- Werbeprospekte,

- Lieferung und Aufstellung,

- Gesamteindruck.

Bei der Wiedergabe ihrer Zufriedenheit gaben wir den interviewten Personen ein Benotungssystem vor $(1=$ sehr gut, 6 = ungenügend).

Die Zufriedenheit mit der Verkaufseinrichtung (beinhaltete 6 Unterkriterien) schwankte zwischen 1,5 (Sauberkeit der Verkaufseinrichtung) und 2,3 (Übersichtlichkeit der Verkaufseinrichtung). Für die Verkaufseinrichtung bekam MEGA-Möbel die Durchschnittsnote 2,0.

Die Besucher gaben MEGA-Möbel bei der Zufriedenheit mit dem Angebot als Durchschnittsnote eine 2,2 (3 Kriterien). Als Sieger dieser drei Kriterien ging die Qualität der Produkte hervor $(2,0)$.

Die beste Bewertung bei der Zufriedenheit mit dem Personal erhielt das Unterkriterium Freundlichkeit des Personals mit 1,8. Die Verfügbarkeit des Personals erhielt nur eine 2,4. Im Durchschnitt bekam diese Kategorie eine 2,1 (3 Unterkriterien).

Die Zufriedenheit mit den Werbeprospekten von MEGAMöbel errang die schlechteste Durchschnittsnote mit 2,3 (3 Unterkriterien) von allen untersuchten Kriterien.

Die Zufriedenheit mit der Lieferung schnitt von allen Kategorien am besten ab. Im Durchschnitt erreichte MEGA-Möbel hier eine 1,8 (3 Unterkriterien).

In der Gesamtzufriedenheit mit MEGA-Möbel gaben die interviewten Personen eine 2,1. Über $75 \%$ der Besucher erteilten ein Gut. 13,8\% gaben ein Befriedigend und 8,3\% ein Sehr gut. Nur 2,0\% der Befragten verteilten eine schlechtere Note als befriedigend.

Darüber hinaus wurden von uns noch weitere Analysen durchgefuihrt. Sie beinhalteten u. a. die Informationsbe-

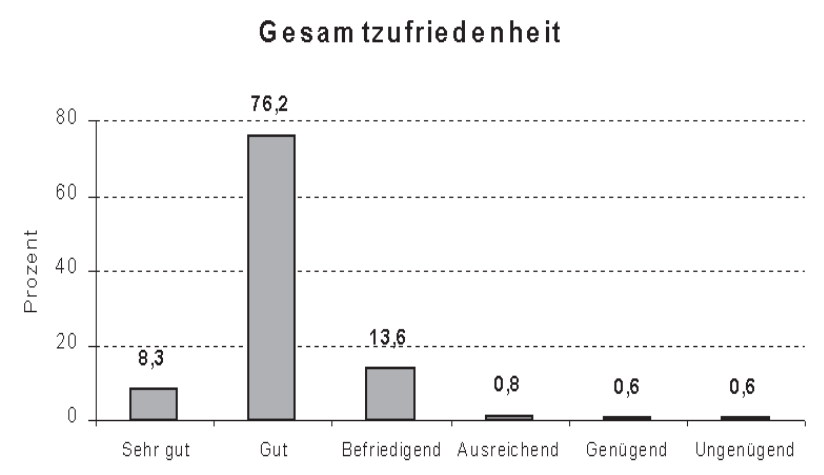

Abb. 4: Gesamtzufriedenheit der MEGA-Möbelbesucher bzw. -kunden

schaffung und die damit verbundene Entscheidungsfindung fuir den Einkauf bei MEGA-Möbel, Einkaufsvolumen in DM, die Verwendung von Prospekten, Erreichungsgrad der Prospekte und Analyse der Wichtigkeit der oben genannten Kriterien für die interviewten Personen.

\section{Fazit}

In der Nachbetrachtung haben wir festgestellt, dass wir den von uns entwickelten Fragebogen vor der eigentlichen Befragung einem Pre-Test hätten unterziehen müssen. Deshalb konnten wir einige Fragen nur sehr schwer beziehungsweise gar nicht auswerten.

In der Zeitdauer fuir ein Interview haben wir uns erheblich verschätzt. Daher haben wir den Fragebogen gestrafft, indem wir die offenen Fragen nur dann ausgefüllt haben, wenn der Kunde von sich aus Informationen herausgab. Insgesamt gesehen war diese Kundenzufriedenheitsanalyse für uns eine gute Möglichkeit, die Theorie in der Praxis umzusetzen (z. B. analytisches Vorgehen bei der Entwicklung des Fragebogens, Vorgehen bei der Befragung, Auswertung der Daten und Präsentation), sowie eine spannende, interessante und teilweise auch stressige Angelegenheit.

\section{Literaturverzeichnis}

Simon, H.: Kundenzufriedenheit. 3., aktualisierte und erweiterte Aufl., Wiesbaden 1998

\section{Autoren}

\section{Michael Jastrob \\ Peter Jentsch}

Stefan Müller

Studenten der Gruppe W 96

Betreuer: Dr. sc. phil. et. Dr. oec. Dieter Heyer

Technische Fachhochschule Wildau

Fachbereich Betriebswirtschaft/Wirtschaftsinformatik Tel. (0 33 75) 508-910 